\title{
SVM based Prediction of Major Histocompatibility Complex Binders: Identification and Analysis of Dracunculus medinensis Peptide
}

\author{
Sonu Mishra ${ }^{1 *}$, Virendra Gomase ${ }^{1}$, and Karbhari V Kale ${ }^{2}$ \\ ${ }^{1}$ Department of Biotechnology, Mewar University, India \\ ${ }^{2}$ Department of Computer Science and IT, Dr.Babasaheb Ambedkar Marathwada University, India \\ Submission: June 06, 2016; Published: June 22, 2016 \\ "Corresponding author: Sonu Mishra, Department of Biotechnology, Mewar University, Chittorgarh, Rajasthan, 312901, India, \\ Email: sonumishra1014@gmail.com; gomase.viren@gmail.com
}

\begin{abstract}
The largest human infecting parasite causes guinea worm disease, known as the disease and cause of poverty due to unavailability of the sanitized water. This is not lethal but causes the long term morbidity and motility in the infected human. In this research work, we predict the peptide binders of antigenic protein from D. medinensis sequence to MHC-I molecules are as 11mer_H2_Db, 10mer_H2_Db, 9mer_H2_Db, 8mer_H2_Db.Also study integrates prediction of peptide MHC class I binding; proteasomal C terminal cleavage and TAP transport efficiency by using sequence and properties of the amino acids. We also found the binding of peptides to different alleles by using Position Specific Scoring Matrix. NADH dehydrogenase subunit 6 (mitochondrion) from D. medinensis is 145 residues long with 137 nonamers having antigenic MHC binding peptides. PSSM based server will predict the peptide binders from D.medinensis of NADH dehydrogenase subunit 6 sequence to MHC-II molecules are as I_Ab.p, I_Ad.p, I_Ag7.p,I_Ak.p which are found antigenic epitopes region in NADH dehydrogenase subunit 6 (mitochondrion) from D. medinensis.
\end{abstract}

Keywords: Antigen; MHC; TAP; PSSM; NADH dehydrogenase subunit 6; Peptide

Abbreviations: MHC: Major Histocompatibility Complex; TAP: Transporter Associated with Antigen Processing; PSSM: Position Specific Scoring Matrices; SVM: Support Vector Machine

\section{Introduction}

The unique life cycle of the D.medinensis takes almost one and half years to complete with unusual six stages. This infection remains asymptomatic during the incubation period. This is one of the largest known human parasites which cause the high rate of the motility and morbidity in infected individuals for long time especially among the school going children's.

The female worm released the larvae after the incubation period. Cyclops (intermediate host) eats infected larvae which gets entry into the human while drinking the contaminated water where the larvae gets mature and complete their six stages of the life cycle and finally the infectious female releases the infectious larvae which induces a painful blister ( 1 to $6 \mathrm{~cm}$ diameter) on the skin of lower limbs; the person develop a slight fever, local skin redness, swelling and severe pruritus around the blister. Other symptoms include diarrhea, nausea, vomiting and dizziness. The severity of the wound infections in the infected individual led to a more complications such as redness and swelling of the skin (cellulitis), boils (abscesses), generalized infection (sepsis), joint infections (septic arthritis) that can cause the joints to lock and deform (contractures), lock jaw (tetanus). The blister burst within 1 to 3 days and female worms one or more slowly comes out from the wounds which causes an excoriating burning sensation and pain .Immersing or pouring water over the blister provide pain reliever. But this the moment that adult female is exposed to the external environment. During emergence of the limbs in open water sources it recognizes the temperature difference and releases the milky white liquid in the water which contains millions of immature larvae, when larvae released in water are ingested by copepods where they mount twice and become infective larvae within two weeks [1-7]. However, Identification of MHC [Major Histocompatibility Complex] binding antigenic peptide molecule will improves the understanding of specificity of immune responses against the pathogen, which is one of the important steps for vaccines discovery.

\section{MHC class I antigen}

The presence of the Major histocompatibility complex class I (MHC-I) molecules has been seen on the on the surface of all nucleated cells and display a large array of peptide epitopes for surveillance by the $\mathrm{CD} 8+\mathrm{T}$ cell repertoire. $\mathrm{CD} 8+\mathrm{T}$ cell responses, 


\section{Organic and Medicinal Chemistry International Journal}

which are essential for the disease or infection control. The CD8 $+\mathrm{T}$ cells are actively and efficiently discriminate between the healthy and the infected cell through the recognition of peptides which are associated with MHC-I molecules present on the cell surface. The peptide with the length range of the 8-11 amino acids, are typically derived from protein antigens in the cytosol that arise from conventional as well as cryptic translational reading frames [8]. Proteins which are classically synthesized in the cytosol undergo proteasomal degradation and the resultant peptides are later on transported into the Endoplasmic Endoplasmic Reticulum (ER) and loaded onto MHC-I molecules [9]. Due to the loading of the peptide the class I MHC stabilizes and pass through to the cell surface where the circulating CD8+ $\mathrm{T}$ cells scans the complexes which is known as 'immune surveillance' [10-19]. Therefore, prediction of TAP binding peptides is important for identification of the MHC class-1 restricted T cell epitopes.

\section{Proteasomal degradation}

Proteasomal degradation is important step in the antigenpresentation process to regulate the balance between intracellular proteins [20]. Inside the proteasome by the action of proteinase the antigenic protein NADH dehydrogenase subunit 6 (mitochondrion) from $D$. medinensis are cleaved into oligopeptides [21] and then these oligopeptides are binds to the TAP, which transports these peptides into the ER.

\section{TAP mediated peptide transport into ER(Endoplasmic Reticulum)}

TAP is heterodimeric transmembrane protein, is a family of $\mathrm{ABC}$ transporter that transports antigenic peptide (NADH dehydrogenase subunit 6 (mitochondrion) from $D$. medinensis into ER [22] because most of the MHC binding peptides are unable to diffuse across membrane, but TAP protein is capable of transporting the peptide inside the ER where it binds to MHC class I molecules. These MHC-peptide complexes will be translocate on the surface of antigen presenting cells [23] and are recognized by T-cell receptors to elicit an immune response.

\section{MHC class II antigen}

The prediction of peptides binding to a MHC class II molecule is difficult due to different side chains and longer length found in the extracellular antigen presentation [24-26]. In the MHC class II antigen presentation process, antigenic protein NADH dehydrogenase subunit 6 (mitochondrion) from D. medinensis are ingested by antigen-presenting cells through the process of endocytosis or phagocytosis, then cleaved by cathepsins a class of protease into oligopeptides in the endosomes, than are fuse with lysosomes containing MHC class II molecules [27] and present them at the cell surface for recognition by $\mathrm{T}$ cells [28-36]. Where $\mathrm{T}$ helper cells trigger an immune response by inflammation and swelling due to phagocytes or may lead to an antibody-mediated immune response via B-cell activation. Since MHCs have a key role in immune system by stimulating cellular and humoral immunity against NADH dehydrogenase subunit 6 (mitochondrion) from D. medinensis and are used for controlling specific immunological processes by creating peptides to bind to specific MHC alleles and this binding affinity to specific peptides are used for designing synthetic peptide vaccines [37-40].

\section{Materials and Methods}

\section{Predictions of MHC class I binding peptide}

MHC binding peptide is predicted using neural networks trained on $\mathrm{C}$ terminals of known epitopes. By using RANKPEP we predict peptide binders to MHCI molecules from protein sequences or sequence alignments using Position Specific Scoring Matrices (PSSMs) whose C terminal end is likely to be the result of proteosomal cleavage.

\section{Prediction of antigenic peptides by cascade SVM based TAPPred method}

By using TAPPred we predict TAP binders on the basis of sequence and the properties of amino acids. We found the MHCI binding regions. The binding affinity of NADH dehydrogenase subunit 6 (mitochondrion) from D.medinensis having 145 amino acids shows 137 nonamers.

\section{Predictions of MHC class II binding peptide}

MHC peptide binding of NADH dehydrogenase subunit 6 (mitochondrion) from D. medinensis predicted using neural networks trained on $\mathrm{C}$ terminals of known epitopes. By using RANKPEP we predict peptide binders to MHCII molecules from protein sequences or sequence alignments using Position Specific Scoring Matrices (PSSMs). MHC molecule binds to some of the peptide fragments generated after proteolytic cleavage of antigen.

\section{Results and Interpretation}

In this research work, we predict the peptide binders of NADH dehydrogenase subunit 6 (mitochondrion) from $D$. medinensis sequence to MHC-I molecules are as 11mer_H2_Db, 10mer_H2_ Db, 9mer_H2_Db, 8mer_H2_Db (Table 2). MHC molecule binds to peptide fragments which are generated after proteolytic cleavage of antigen tend to be high-efficiency binders. TAP is an important transporter that involved in the translocation of peptides from cytosol to ER. TAP binds and translocate selective peptides for binding to specific MHC molecules. Therefore, predicting binding affinity of those peptides toward the TAP transporter is crucial to identify the MHC class-1 restricted T cell epitopes. Cascade based support vector machine shows 42 High affinity TAP binder residues at $\mathrm{N}$ and $\mathrm{C}$ termini using sequence and properties of the amino acids of NADH dehydrogenase subunit 6 (mitochondrion) from D.medinensis (Table 3). This method integrates prediction of peptide MHC class I binding; proteasomal C terminal cleavage and TAP transport efficiency by using sequence and properties of the amino acids. We also found the binding of peptides to different alleles by using Position Specific Scoring Matrix. NADH dehydrogenase subunit 6 (mitochondrion) from $D$. medinensis is 145 residues long with 137 nonamers having antigenic MHC 


\section{Organic and Medicinal Chemistry International Journal}

binding peptides. PSSM based server will predict the peptide I_Ad.p,I_Ag7, I_Ak which are found antigenic epitopes region binders of $\mathrm{NADH}$ dehydrogenase subunit 6 (mitochondrion) in NADH dehydrogenase subunit 6 (mitochondrion) from $D$. from D. medinensis sequence to MHCII molecules are as I_Ab.p, medinensis (Table 1).

Table 1: Peptide binders of NADH dehydrogenase subunit 6 (mitochondrion) from D. medinensis to MHC-II molecules.

\begin{tabular}{|c|c|c|c|c|c|c|c|c|}
\hline MHC-II Allele & RANK & POS. & N & SEQUENCE & C & MW (Da) & SCORE & \% OPT. \\
\hline I_Ab & 1 & 47 & LYV & WYSYYVCLL & FLS & 1168.42 & 12.487 & $35.04 \%$ \\
\hline I_Ab & 2 & 17 & SMS & YFSFDPMKS & ALF & 1103.27 & 10.081 & $28.29 \%$ \\
\hline I_Ab & 3 & 96 & CMS & FDLYDYGGM & GLG & 1062.17 & 8.903 & $24.99 \%$ \\
\hline I_Ab & 4 & 48 & YVW & YSYYVCLLF & LSG & 1152.39 & 7.98 & $22.40 \%$ \\
\hline I_Ab & 5 & 119 & VYY & FWLVGVLLF & YLN & 1052.36 & 7.618 & $21.38 \%$ \\
\hline I_Ad & 1 & 50 & WYS & YYVCLLFLS & GIF & 1102.37 & 9.332 & $17.56 \%$ \\
\hline I_Ad & 2 & 66 & IIV & YFSSIGFCE & VFS & 1034.17 & 7.484 & $14.08 \%$ \\
\hline I_Ad & 3 & 61 & SGI & FVIIVYFSS & IGF & 1056.28 & 6.352 & $11.95 \%$ \\
\hline I_Ad & 4 & 22 & SFD & PMKSSALFLV & FSL & 987.27 & 4.957 & $9.33 \%$ \\
\hline I_Ad & 5 & 9 & VLV & SMLFASMSY & FSF & 1018.22 & 3.374 & $6.35 \%$ \\
\hline I_Ag7 & 1 & 120 & YYF & WLVGVLLFY & LNF & 1068.36 & 8.163 & $19.97 \%$ \\
\hline I_Ag7 & 2 & 137 & SFL & TFMGALRSF & & 1011.21 & 7.908 & $19.35 \%$ \\
\hline I_Ag7 & 3 & 94 & FFC & MSFDLYDYG & GMG & 1092.2 & 6.486 & $15.87 \%$ \\
\hline I_Ag7 & 4 & 49 & VWY & SYYVCLLFL & SGI & 1102.37 & 6.209 & $15.19 \%$ \\
\hline I_Ag7 & 5 & 38 & GLL & PVISCNLYV & WYS & 989.2 & 6.128 & $14.99 \%$ \\
\hline I_Ak & 1 & 79 & FSL & DFFSFLLCV & YFS & 1072.3 & 17.533 & $43.94 \%$ \\
\hline I_Ak & 2 & 43 & ISC & NLYVWYSYY & VCL & 1229.4 & 9.359 & $23.46 \%$ \\
\hline I_Ak & 3 & 121 & YFW & LVGVLLFYL & NFI & 1018.31 & 8.731 & $21.88 \%$ \\
\hline I_Ak & 4 & 59 & FLS & GIFVIIVYF & SSI & 1052.33 & 8.001 & $20.05 \%$ \\
\hline I_Ak & 5 & 100 & DLY & DYGGMGLGL & VVS & 863.98 & 7.069 & $17.72 \%$ \\
\hline
\end{tabular}

Matrix: I_Ab.p.mtx, Consensus: YYAPWCNNA, Optimal Score: 35.632, Binding Threshold: 9.52; Matrix: I_Ad.p.mtx, Consensus: QMVHAAHAE, Optimal Score: 53.145, Binding Threshold: 7.10; Matrix: I_Ag7.p.mtx, Consensus: WYAHAFKYV, Optimal Score: 40.873.

Binding Threshold: 7.54; Matrix: I_Ak.p.mtx, Consensus: DFWCWECCC, Optimal Score: 39.9, Binding Threshold: 14.17). (All rows highlighted in red represent predicted binders \& A peptide highlighted in violet has a C-teminus predicted by the cleavage model used).

Table 2: Peptide binders of NADH dehydrogenase subunit 6 (mitochondrion) from D. medinensis to MHC-I molecules, having C-terminal ends are proteosomal cleavage sites.

\begin{tabular}{|l|c|c|c|c|c|c|c|c|}
\hline MHC-I Allele & RANK & POS. & N & SEQUENCE & C & MW (Da) & SCORE & \% OPT. \\
\hline 8mer_H2_Db & 1 & 68 & VYF & SSIGFCEV & FSL & 822.94 & 22.661 & $43.17 \%$ \\
\hline 8mer_H2_Db & 2 & 38 & GLL & PVISCNLY & VWY & 890.07 & 12.523 & $23.86 \%$ \\
\hline 8mer_H2_Db & 3 & 57 & LLF & LSGIFVII & VYF & 843.08 & 8.163 & $15.55 \%$ \\
\hline 8mer_H2_Db & 4 & 76 & CEV & FSLDFFSF & LLC & 991.13 & 8.108 & $15.45 \%$ \\
\hline 8mer_H2_Db & 5 & 45 & CNL & YVWYSYYV & CLL & 1101.27 & 7.867 & $14.99 \%$ \\
\hline 8mer_H2_Db & 6 & 55 & VCL & LFLSGIFV & IIV & 877.1 & 5.124 & $9.76 \%$ \\
\hline 8mer_H2_Db & 7 & 103 & DYG & GMGLGLVV & SDM & 726.92 & 4.278 & $8.15 \%$ \\
\hline 8mer_H2_Db & 8 & 82 & DFF & SFLLCVYF & SFF & 973.21 & 3.602 & $6.86 \%$ \\
\hline 8mer_H2_Db & 9 & 109 & LGL & VVSDMYLV & YYF & 907.09 & 2.083 & $3.97 \%$ \\
\hline 8mer_H2_Db & 10 & 94 & FFC & MSFDLYDY & GGM & 1035.15 & 1.865 & $3.55 \%$ \\
\hline 9mer_H2_Db & 1 & 86 & FLL & CVYFSFFCM & SFD & 1128.4 & 19.178 & $38.08 \%$ \\
\hline 9mer_H2_Db & 2 & 15 & FAS & MSYFSFDPM & KSA & 1106.29 & 17.399 & $34.55 \%$ \\
\hline 9mer_H2_Db & 3 & 68 & VYF & SSIGFCEVF & SLD & 970.12 & 12.268 & $24.36 \%$ \\
\hline 9mer_H2_Db & 4 & 76 & CEV & FSLDFFSFL & LCV & 1104.29 & 11.102 & $22.04 \%$ \\
\hline 9mer_H2_Db & 5 & 67 & IVY & FSSIGFCEV & FSL & 970.12 & 9.504 & $18.87 \%$ \\
\hline 9mer_H2_Db & 6 & 131 & YLN & FISSFLTFM & GAL & 1074.31 & 8.837 & $17.55 \%$ \\
\hline
\end{tabular}




\section{Organic and Medicinal Chemistry International Journal}

\begin{tabular}{|c|c|c|c|c|c|c|c|c|}
\hline 9mer_H2_Db & 7 & 81 & LDF & FSFLLCVYF & SFF & 1120.39 & 8.767 & $17.41 \%$ \\
\hline 9mer_H2_Db & 8 & 121 & YFW & LVGVLLFYL & NFI & 1018.31 & 8.298 & $16.48 \%$ \\
\hline 9mer_H2_Db & 9 & 73 & IGF & CEVFSLDFF & SFL & 1088.26 & 7.597 & $15.08 \%$ \\
\hline 9mer_H2_Db & 10 & 83 & FFS & FLLCVYFSF & FCM & 1120.39 & 6.513 & $12.93 \%$ \\
\hline 10mer_H2_Db & 1 & 25 & PMK & SALFLVFSLL & GLL & 1091.37 & 9.355 & $15.89 \%$ \\
\hline 10mer_H2_Db & 2 & 130 & FYL & NFISSFLTFM & GAL & 1188.41 & 8.663 & $14.72 \%$ \\
\hline 10mer_H2_Db & 3 & 18 & MSY & FSFDPMKSAL & FLV & 1124.33 & 8.113 & $13.78 \%$ \\
\hline 10mer_H2_Db & 4 & 58 & LFL & SGIFVIIVYF & SSI & 1139.41 & 6.333 & $10.76 \%$ \\
\hline 10mer_H2_Db & 5 & 34 & FSL & LGLLPVISCN & LYV & 1010.26 & 6.237 & $10.60 \%$ \\
\hline 10mer_H2_Db & 6 & 42 & VIS & CNLYVWYSYY & VCL & 1332.54 & 5.48 & $9.31 \%$ \\
\hline 10mer_H2_Db & 7 & 45 & $\mathrm{CNL}$ & YVWYSYYVCL & LFL & 1317.57 & 4.949 & $8.41 \%$ \\
\hline 10mer_H2_Db & 8 & 40 & LPV & ISCNLYVWYS & YYV & 1206.42 & 4.116 & $6.99 \%$ \\
\hline 10mer_H2_Db & 9 & 93 & SFF & CMSFDLYDYG & GMG & 1195.34 & 2.565 & $4.36 \%$ \\
\hline 10mer_H2_Db & 10 & 85 & SFL & LCVYFSFFCM & SFD & 1241.56 & 2.081 & $3.54 \%$ \\
\hline 11mer_H2_Db & 1 & 96 & CMS & FDLYDYGGMGL & GLV & 1232.38 & 15.09 & $18.98 \%$ \\
\hline 11mer_H2_Db & 2 & 122 & FWL & VGVLLFYLNFI & SSF & 1279.59 & 13.674 & $17.20 \%$ \\
\hline 11mer_H2_Db & 3 & 44 & SCN & LYVWYSYYVCL & LFL & 1430.73 & 13.575 & $17.08 \%$ \\
\hline 11mer_H2_Db & 4 & 42 & VIS & CNLYVWYSYYV & CLL & 1431.67 & 11.283 & $14.19 \%$ \\
\hline 11mer_H2_Db & 5 & 45 & CNL & YVWYSYYVCLL & FLS & 1430.73 & 9.818 & $12.35 \%$ \\
\hline 11mer_H2_Db & 6 & 62 & GIF & VIIVYFSSIGF & CEV & 1226.49 & 8.649 & $10.88 \%$ \\
\hline 11mer_H2_Db & 7 & 85 & SFL & LCVYFSFFCMS & FDL & 1328.64 & 7.987 & $10.05 \%$ \\
\hline 11mer_H2_Db & 8 & 93 & SFF & CMSFDLYDYGG & MGL & 1252.39 & 7.881 & $9.91 \%$ \\
\hline 11mer_H2_Db & 9 & 84 & FSF & LLCVYFSFFCM & SFD & 1354.72 & 7.856 & $9.88 \%$ \\
\hline 11mer_H2_Db & 10 & 107 & MGL & GLVVSDMYLVY & YFW & 1240.48 & 7.436 & $9.35 \%$ \\
\hline
\end{tabular}

Matrix: 8mer H2 Db.p.mtx; Consensus: QNWNCCTI; Optimal Score: 52.494; Matrix: 9mer H2 Db.p.mtx; Consensus: FCIHNCDYM; Optimal Score: 50.365; Binding Threshold: 17.96; Matrix: 10mer_H2_Kd.p.mtx; Consensus: WYPPPGKTTL; Optimal Score: 44.281; Binding Threshold: 21.13; Binding Threshold: 33.04;

Matrix: 11mer_H2_Db.p.mtx, Consensus: CGVYNFYYCCY, Optimal Score: 79.495, Binding Threshold: 56.96. (All rows highlighted in red represent predicted binders \& A peptide highlighted in violet has a $\mathrm{C}$-teminus predicted by the cleavage model used).

Table 3: Cascade SVM based High affinity TAP Binders of NADH dehydrogenase subunit 6 (mitochondrion) proteins from $D$. medinensis.

\begin{tabular}{|c|c|c|c|c|}
\hline Peptide Rank & Start Position & Sequence & Score & Predicted Affinity \\
\hline 1 & 83 & FLLCVYFSF & 8.641 & High \\
\hline 2 & 70 & IGFCEVFSL & 8.64 & High \\
\hline 3 & 50 & YYVCLLFLS & 8.637 & High \\
\hline 4 & 131 & FISSFLTFM & 8.635 & High \\
\hline 5 & 24 & KSALFLVFS & 8.621 & High \\
\hline 6 & 92 & FCMSFDLYD & 8.615 & High \\
\hline 7 & 128 & YLNFISSFL & 8.604 & High \\
\hline 8 & 76 & FSLDFFSFL & 8.594 & High \\
\hline 9 & 22 & PMKSALFLV & 8.545 & High \\
\hline 10 & 53 & CLLFLSGIF & 8.538 & High \\
\hline 11 & 95 & SFDLYDYGG & 8.514 & High \\
\hline 12 & 61 & FVIIVYFSS & 8.475 & High \\
\hline 13 & 57 & LSGIFVIIV & 8.468 & High \\
\hline 15 & 14 & SMSYFSFDP & 8.463 & High \\
\hline 16 & 135 & FLTFMGALR & 8.439 & 8.423 \\
\hline
\end{tabular}




\section{Organic and Medicinal Chemistry International Journal}

\begin{tabular}{|c|c|c|c|c|}
\hline 17 & 36 & LLPVISCNL & 8.421 & High \\
\hline 18 & 72 & FCEVFSLDF & 8.388 & High \\
\hline 19 & 41 & SCNLYVWYS & 8.352 & High \\
\hline 20 & 1 & MLFFFVLVS & 8.267 & High \\
\hline 21 & 34 & LGLLPVISC & 8.233 & High \\
\hline 22 & 51 & YVCLLFLSG & 8.215 & High \\
\hline 23 & 66 & YFSSIGFCE & 8.171 & High \\
\hline 24 & 94 & MSFDLYDYG & 7.945 & High \\
\hline 25 & 17 & YFSFDPMKS & 7.704 & High \\
\hline 26 & 56 & FLSGIFVII & 7.654 & High \\
\hline 27 & 21 & DPMKSALFL & 7.542 & High \\
\hline 28 & 87 & VYFSFFCMS & 7.537 & High \\
\hline 29 & 124 & VLLFYLNFI & 7.481 & High \\
\hline 30 & 62 & VIIVYFSSI & 7.476 & High \\
\hline 31 & 102 & GGMGLGLVV & 7.172 & High \\
\hline 32 & 27 & LFLVFSLLG & 7.113 & High \\
\hline 33 & 38 & PVISCNLYV & 7.045 & High \\
\hline 34 & 32 & SLLGLLPVI & 6.758 & High \\
\hline 35 & 125 & LLFYLNFIS & 6.681 & High \\
\hline 36 & 6 & VLVSMLFAS & 6.513 & High \\
\hline 37 & 106 & LGLVVSDMY & 6.43 & High \\
\hline 38 & 31 & FSLLGLLPV & 6.377 & High \\
\hline 39 & 15 & MSYFSFDPM & 6.281 & High \\
\hline 40 & 16 & SYFSFDPMK & 6.158 & High \\
\hline 41 & 82 & SFLLCVYFS & 6.05 & High \\
\hline 42 & 5 & FVLVSMLFA & 6.007 & High \\
\hline
\end{tabular}

* TAPPred showing Cascade SVM based High affinity TAP Binders sites, their sequence, rank, position and scores are displayed in the tabular output are to be found 15 High affinity TAP Transporter peptide regions which represents predicted TAP binders residues which occur at $\mathrm{N}$ and $\mathrm{C}$ termini NADH dehydrogenase subunit 6 (mitochondrion) from $D$. medinensis.

* The RANKPEP consists of a list of selected peptides binding potential (score) to the MHC molecule from the query given at a selected threshold. Peptides shown here contain a C-terminal residue that is predicted to be the result of proteasomal cleavage and also focus on the prediction of conserved epitopes that help to avoid immune evasion resulting from mutation. Proteasomal cleavage options are only applied to the prediction of MHCIrestricted peptides.

* TAPPred showing Cascade SVM based High affinity TAP Binders sites, their sequence, rank, position and scores are displayed in the tabular output are to be found 42 High affinity TAP Transporter peptide regions which represents predicted TAP binders residues which occur at $\mathrm{N}$ and $\mathrm{C}$ termini NADH dehydrogenase subunit 6 (mitochondrion) from $D$. medinensis.

\section{Conclusion}

$\mathrm{NADH}$ dehydrogenase subunit 6 (mitochondrion) an antigenic proteins from $D$. medinensis involved multiple antigenic components to direct and empower the immune system to protect the host from the pathogenesis. Major histocompatibility complexes I and II (MHC-I and MHC-II) display specificity to

bind with their respective epitopes. MHC class molecules are cell surface proteins that take active part in host immune reactions to response for almost all antigens. This knowledge of the immune responses to an antigen protein NADH dehydrogenase subunit 6 (mitochondrion) from D. medinensis clear that the whole protein is not necessary for raising the immune response, but a small fragment of antigen can induce immune response against whole antigen. This means the increase in affinity of MHC binding peptides may result in enhancement of immunogenicity of NADH dehydrogenase subunit 6 (mitochondrion) from $D$. medinensis hence are helpful in silico to design and develop highly predictive computational tools for the identification of T-cell epitopes. Finally, accurate prediction remains vital for the future to design synthetic peptide vaccine.

\section{References}

1. Mullner A, Helfer A, KotlyarD, Oswald J, EfferthT (2011) Chemistry and pharmacology of neglected helminthic disease. Curr Med Chem 18(5): 767-789.

2. Ruiz-Tiben E, Hopkins DR (2006) Dracunculiasis (Guinea worm disease) eradication. Adv Parasitol 61: 275-309. 


\section{Organic and Medicinal Chemistry International Journal}

3. IriemenamNC, Oyibo WA, Fagbenro-Beyioku AF (2008) Dracunculiasis -The saddle is virtually ended. Parasitol Res 102(3): 343-347.

4. Silkjaer T, Nyvold CG, Juhl-Christensen C, Hokland P, Nørgaard JM (2013) Mitochondrial cytochrome c oxidase subunit II variations predict adverse prognosis in cytogenetically normal acute myeloid leukaemia. Eur J Haematol 91(4): 295-303.

5. Hamblet NS, Ragland B, Ali M, Conyers B, Castora FJ (2006) Mutations in mitochondrial-encoded cytochrome c oxidase subunits I, II, and III genes detected in Alzheimer's disease using single-strand conformation polymorphism. Electrophoresis 27(2): 398-408.

6. Nwoke BE (1992) Behavioral aspects and their possible uses in the control of dracunliasis (guinea-worm) in Igwun river basin area of Imo State, Nigeria. Angew Parasitol 34(4): 205-210.

7. Muller R (1985) Life cycle of DracunculusMedinesis. In workshop on opportunities for control of dracunculiasis : contaminated papers, washinton, DC: National Academy Press.

8. Shastri N, Schwab S, Serwold T (2002) Producing nature's gene-chips: the generation of peptides for display by MHC class I molecules. Annu Rev Immunol 20: 463-493.

9. Blum J, Wearsch P, Cresswell P (2013) Pathways of antigen processing Annu Rev Immunol 31: 443-473.

10. Rock K, Gramm C, Rothstein L, Clark, Stein R, et al. (1994) Inhibitors of the proteasome block the degradation of most cell proteins and the generation of peptides presented on MHC class I molecules. Cell 78(5): 761-771.

11. Bhasin M, Raghava GP (2005) Pcleavage: an SVM based method for prediction of constitutive proteasome and immunoproteasome cleavage sites in antigenic sequences. Nucleic Acids Res 33: W202-207.

12. Vyas JM, Vander Veen AG, Ploegh HL (2008) The known unknowns of antigen processing and presentation. Nat Rev Immunol 8(8): 607-618.

13. Kelly A, Powis SH, Kerr LA, Mockridge I, Elliott T, et al. (1992) Assembly and function of the two $\mathrm{ABC}$ transporter proteins encoded in the human major histocompatibility complex. Nature 355(6361): 641-644

14. Lautscham G, Rickinson A, Blake N (2003) TAP-independent antigen presentation on MHC class I molecules: Lessons from Epstein-Barr virus. Microbes and Infection 5(4): 291-299.

15. Nussbaum AK, Kuttler C, Tenzer S, Schild H (2003) Using the World Wide Web for predicting CTL epitopes. Curr Opin Immunol 15(1): 6974 .

16. Lankat-Buttgereit B, Tampe R (2002) The transporter associated with antigen processing: Function and implications in human diseases. Physiol Rev 82(1): 187-204.

17. Rock KL, York IA, Goldberg AL (2004) Post-proteasomal antigen processing for major histocompatibility complex class I presentation. Nature immunology 5(7): 670-677.

18. Hammerling GJ, Vogt AB, Kropshofer H (1999) Antigen processing and presentation: Towards the millennium. Immunol Rev 172: 5-11.

19. Bhasin M, Ragahava GPS (2007) A hybrid approach for predicting promiscuous MHC class I restricted T cell epitopes. J Biosciences 32(1): $31-42$.

20. Brussic V, Bajic VB, Petrovsky N (2004) Computational methods for prediction of T-cell epitopes-a framework for modelling, testing and applications. Methods 34(4): 436-443.

21. Reidesel H, Kolbeck B, Schmetzer O, Knapp EW (2004) Peptide binding at class I major histocompatibility complex scored with linear functions and support vector machines. Genome Informatics 15(1): 198-212.
22. Princiotta MF, Finzi D, Qian SB, Gibbs J, Schuchmann S, et al. (2003) Quantitating protein synthesis, degradation and endogenous antigen processing. Immunity 18(3): 343-354.

23. Rock KL, York IA, Goldberg AL (2004) Post-proteasomal antigen processing for major histocompatibility complex class I presentation. Nat Immunol 5(7): 670-677.

24. Procko E, Gaudet R (2009) Antigen processing and presentation: TAPping into ABC transporters. Curr Opin Immunol 21(1): 84-91.

25. Yewdell JW, Reits E, Neefjes J ( 2003) Making sense of mass destruction: quantitating MHC class I antigen presentation. Nat Rev Immunol 3(12): 952-961.

26. Stern LJ, Wiley DC (1994) Antigenic peptide binding by class I and class II histocompatibility proteins. Behring Inst Mitt (94):1-10.

27. Hammer J, Bono E, Gallazzi F, Belunis C, Nagy Z, et al. (1994) Precise prediction of major histocompatibility complex class II-peptide interaction based on peptide side chain scanning. J Exp Med 180(6): 2353-2358.

28. Jardetzky TS, Brown JH, Gorga JC, Stern LJ, Urban RG, et al. (1996) Crystallographic analysis of endogenous peptides associated with HLA-DR1 suggests a common, polyproline II-like conformation for bound peptides. Proc Natl Acad Sci USA 93(2): 734-738.

29. Cresswell P (1994) Assembly, transport, and function of MHC class Il molecules. Annu Rev Immunol 12: 259-293.

30. Rudolph MG, Stanfield RL, Wilson IA (2006) How TCRs bind MHCs, peptides, and coreceptors. Annu Rev Immunol 24: 419-466.

31. Nielsen M, Lund O, Buus S, Lundegaard C ( 2010) MHC Class II epitope predictive algorithms. Immunology 130(3): 319-328.

32. Nielsen M, Lundegaard C, Worning P, Hvid CS, Lamberth K, et al. (2004) Improved prediction of MHC class I and class II epitopes using a novel Gibbs sampling approach. Bioinformatics 20(9): 1388-1397.

33. Murugan N, Dai Y (2005) Prediction of MHC class II binding peptides based on an iterative learning model. Immunome Res 1: 6 .

34. Salomon J, Flower DR (2006) Predicting Class II MHC-Peptide binding: a kernel based approach using similarity scores. BMC Bioinformatics 7: 501 .

35. Bordner AJ, Mittelmann HD (2010) Prediction of the binding affinities of peptides to class II MHC using a regularized thermodynamic model. BMC Bioinformatics 11: 41

36. Nielsen M, Lundegaard C, Lund O (2007) Prediction of MHC class II binding affinity using SMM-align, a novel stabilization matrix alignment method. BMC Bioinformatics 8: 238.

37. Gomase VS, Chitlange NR (2012) Sensitive Quantitative Predictions of MHC Binding Peptides and Fragment Based Peptide Vaccines from Taeniacrassiceps. J Vaccines Vaccin 3: 131.

38. Changbhale SS, Chitlange NR, Gomase VS, Kale KV (2012) An Immunoinformatics Approach to Design Synthetic Peptide Vaccine from Dendroaspispolylepispolylepis Dendrotoxin-K(DTX-K). Journal of Environmental \& Analytical Toxicology 2(7): 157.

39. Reche PA, Glutting JP, Zhang H, Reinherz EL ( 2004) Enhancement to the RANKPEP resource for the prediction of peptide binding to MHC molecules using profiles. Immunogenetics 56(6): 405-419.

40. Gomase VS, Chitlange NR, Changbhale SS, Kale KV (2013) Prediction of Brugiamalayi antigenic peptides: candidates for synthetic vaccine design against lymphatic filariasis. Protein Pept Lett 20(8): 864-887. 